\title{
Efficacy of the Combination of Voriconazole and Caspofungin in Experimental Pulmonary Aspergillosis by Different Aspergillus Species
}

\author{
Ming Zhang $\cdot$ Xin Su $\cdot$ Wen-Kui Sun • \\ Fei Chen $\cdot$ Xiao-Yong Xu $\cdot$ Yi Shi
}

Received: 2 September 2013/ Accepted: 25 November 2013/Published online: 6 December 2013

(C) The Author(s) 2013. This article is published with open access at Springerlink.com

\begin{abstract}
Objectives Invasive pulmonary aspergillosis (IPA) caused by Aspergillus fumigatus, Aspergillus flavus, or Aspergillus niger is associated with high mortality. We evaluated the efficacy and compared the therapeutic effect differences of voriconazole (VRC) in combination with caspofungin (CAS) in transiently neutropenic rats infected by A. fumigatus, A. flavus, or A. niger.

Methods Treatment groups consisted of VRC (10 mg/kg q12 h) monotherapy, CAS (1 mg/kg/day) monotherapy, combination of VRC $(10 \mathrm{mg} / \mathrm{kg}$ $\mathrm{q} 12 \mathrm{~h})+\mathrm{CAS}(1 \mathrm{mg} / \mathrm{kg} /$ day $)$, and no drug for 10 consecutive days. The efficacy and the difference in the treatments were evaluated through prolongation of survival, reduction in serum galactomannan levels and residual fungal burden, and histological studies.

Results For all the strains, the combination of VRC and CAS led to significant prolongation in survival $(P<0.05)$ and reduction in residual fungal burden $(P<0.05)$ compared with CAS alone, and decrease in serum galactomannan levels $(P<0.05)$ compared with either agent alone. The survival in the combined therapy groups was significantly improved compared
\end{abstract}

M. Zhang $\cdot$ X. Su $\cdot$ W.-K. Sun $\cdot$ F. Chen .

X.-Y. Xu · Y. Shi $(\square)$

Department of Respiratory and Critical Care Medicine, Jinling Hospital, Nanjing University School of Medicine, 305 East Zhongshan Road, Nanjing 210002, China e-mail: shiyi56@126.com to VRC monotherapy for the strains of A. flavus and $A$. niger $(P<0.05)$, but no significant difference for the strains of A. fumigatus $(P>0.05)$.

Conclusions Combination of VRC and CAS was synergistic in IPA by A. flavus and A. niger, but small efficacy benefits in IPA by A. fumigatus.

Keywords Voriconazole - Caspofungin · Combination · Aspergillus fumigatus . Aspergillus flavus $\cdot$ Aspergillus niger

\section{Introduction}

Invasive aspergillosis (IA) is an opportunistic infection caused by the fungi of the genus Aspergillus. Over $90 \%$ of IA cases involve the lung, leading to invasive pulmonary aspergillosis (IPA) [1]. Aspergillus fumigatus is the most common species recovered from cases of IPA [2], followed by Aspergillus flavus and Aspergillus niger. Liking with A. fumigatus, IPA caused by A. flavus or A. niger is also associated with high mortality rates [3].

Voriconazole (VRC) is considered the primary therapy for IPA, based on the results of randomized clinical trials [4, 5], alternatives being liposomal amphotericin B (L-AMB), amphotericin B lipid complex (ABLC), caspofungin (CAS), micafungin, posaconazole, and itraconazole. Despite these treatment options, the outcomes of IPA remain poor, with 
mortality rates of 25-35\% 12 weeks after diagnosis [6]. Owing to the target of triazoles at cell membrane and echinocandins at cell wall [4], the combination therapy of azoles and echinocandins may result in synergistic interaction against Aspergillus spp, including a wider spectrum of efficacy, lowered toxicity, and prevented the emergence of resistance [7-9]. However, some studies showed that the combination of azole and echinocandin could not significantly improve the therapeutic outcome [10], even may be potentially antagonistic [11].

There are some reports of combination therapies of azole and echinocandin in the treatment for IA infected by A. fumigatus [7-14],more rarely by $A$. flavus [15] and A. niger. In this study, we established the model of IPA in transiently neutropenic rats infected by A. fumigatus, A. flavus, or A. niger. The dosage of VRC and CAS was adjusted according to the pharmacokinetic/pharmacodynamic

(PK/PD) described in the previous studies [10, 12, 16-18]. The therapeutic efficacy and difference in both agents in every strain, administered alone or in combination, were examined.

\section{Materials and Methods}

Three clinical isolates of A. fumigatus, A. flavus, and A. niger obtained from patients with proven IPA in Jinling hospital were used in this study. The isolates had been stored in $10 \%$ glycerol broth at $-80{ }^{\circ} \mathrm{C}$. To prepare the inocula, the isolates were cultivated on Sabouraud dextrose agar (SDA) at $35{ }^{\circ} \mathrm{C}$ for 5 days and cultures were then suspended in sterile $0.1 \%$ Triton 80 in phosphate-buffered saline (PBS) and filtered through sterile gauze to remove hyphae. The resulting suspensions were adjusted to the desired concentration of $1 \times 10^{9}$ conidia/mL in sterile PBS by counting with a hemacytometer. The conidial suspension was used within $24 \mathrm{~h}$ and stored at $4{ }^{\circ} \mathrm{C}$.

\section{In Vitro Studies}

The in vitro antifungal susceptibility test of the three strains to VRC (Vfend, Pfizer Inc. Madrid, Spain) and CAS (Cancidas, Merck Sharp \& Dohme Pty. Ltd. New South Wales, Australia) was performed in triplicate according to the Clinical and Laboratory Standards
Institute (CLSI) standard M38-A2 microdilution methods [19]. For VRC, the minimal inhibitory concentration (MIC) was determined, and for CAS, the minimal effective concentration (MEC) was determined. The interactions of the combination of VRC and CAS were evaluated by determining the fractional concentration index (FICI) using a checkerboard method [20]. MIC end points were determined as MIC-0 (100\% of growth inhibition) of VRC alone and in combination with CAS. The FICI was defined as $\mathrm{FICI}=(\mathrm{Ac} / \mathrm{Aa})+(\mathrm{Bc} / \mathrm{Ba})$, where $\mathrm{Ac}$ and $\mathrm{Bc}$ are the MICs of VRC and CAS in combination, Aa is the MIC of VRC, and $\mathrm{Ba}$ is the MEC of CAS, as previously described [21-23]. Drug interactions were classified as synergistic $(\mathrm{FICI} \leq 0.5)$, indifferent $(\mathrm{FICI}>0.5$ but $\leq 4)$, or antagonistic (FICI $>4$ ) [24].

Animal Model

A total of 180 male Sprague-Dawley rats, aged 6-8 weeks, weight $220-250 \mathrm{~g}$, were used in these experiments. All the animals were housed under standard conditions and allowed ad libitum access to food and water. This study was conducted in conformity with institutional guidelines for the care and use of laboratory animals in Jinling Hospital, Nanjing, China, and performed according to the National Institutes of Health Guide for Care and Use of Laboratory Animals. Pulmonary aspergillosis was established as described elsewhere [25]. The animals were immunosuppressed with cyclophosphamide (75 mg/kg of body weight intraperitoneally twice) plus Methylprednisolone (13 mg/kg intramuscularly once) 2 days before infection. And the animals received Levofloxacin (Cravit; $10 \mathrm{mg} / \mathrm{kg}$ intravenous injection daily) from day 2 preinfection to day 10 after challenge to prevent bacterial infections. Rats were intratracheally inoculated with a single administration of $1 \times 10^{8}$ conidia of A. fumigatus, A. flavus, or A. niger in $100 \mu \mathrm{l}$ of sterile PBS [26].

\section{Antifungal Treatment}

The efficacy of combination therapy using VRC and CAS was assessed in this study. For each strain and each treatment, 180 animals were randomized into groups of 15 rats. Ten rats were assigned randomly to the survival study and five rats for residual fungal burden and 
histopathological studies. Rats were grouped to receive VRC $10 \mathrm{mg} / \mathrm{kg} \mathrm{q} 12 \mathrm{~h}$ intravenously, CAS $1 \mathrm{mg} / \mathrm{kg} /$ day intravenously, a combination of $\operatorname{VRC}(10 \mathrm{mg} / \mathrm{kg}$ q12 h intravenously)and CAS (1 mg/kg/day intravenously), or no drug (untreated controls). All treatments began 1 day post-infection and continued for 10 days.

\section{Survival Study}

Ten rats were assigned randomly to the survival study. The survival time after infection was recorded daily for 15 days.

\section{Galactomannan Assays}

Blood from each rat was collected every other day to determine serum galactomannan levels. Serum galactomannan concentrations were performed by the Platelia Aspergillus EIA (Platelia Aspergillus; Sanofi Diagnostics, Marnes-La Coquette, France) according to the manufacturer's instructions. Values of EIA were expressed as galactomannan index (GI) plotted over time.

\section{Residual Fungal Burden}

The rats were killed on day 5 after challenge. Each right lung, weighed individually, placed $(\mathrm{W}: \mathrm{V}=1: 1)$ in sterile $0.1 \%$ Triton 80 in PBS, and homogenized in a tissue homogenizer. Serial tenfold dilutions of the homogenates were cultured for $48 \mathrm{~h}$ on SDA plates at $35^{\circ} \mathrm{C}$. Fungal burden was expressed as $\log 10 \mathrm{CFU} / \mathrm{g}$ of lung tissue.

Histopathological Study

The left lungs of the killed rats were excised and fixed in $10 \%$ buffered formalin. Paraffin-embedded tissue sections were then sectioned and stained with hematoxylin-eosin (HE).

\section{Statistical Analysis}

Statistical evaluation of survival was done by the Kaplan-Meier analysis. Differences in rat survival rates were assessed by the log rank test. Galactomannan serum levels and fungal burden data were assessed by the Mann-Whitney U test. A $P$ value of $\leq 0.05$ was considered to be statistically significant.
Table 1 In vitro antifungal activity of voriconazole and caspofungin against three strains of A. fumigatus, A. flavus, and A. niger

\begin{tabular}{llll}
\hline Strain & MIC-VRC $(\mu \mathrm{g} / \mathrm{ml})$ & MEC-CAS $(\mu \mathrm{g} / \mathrm{ml})$ & FICI \\
\hline A. fumigatus & 0.5 & 0.06 & 0.50 \\
A. flavus & 0.25 & 0.06 & 0.38 \\
A. niger & 0.25 & 0.03 & 0.25 \\
\hline
\end{tabular}

$V R C$ voriconazole, $C A S$ caspofungin, $M I C$ minimal inhibitory concentration, $M E C$ minimal effective concentration, FICI fractional inhibitory concentration index

\section{Results}

In Vitro Studies

The in vitro antifungal activity of VOR and CAS alone or in combination against the three strains of Aspergillus is shown in Table 1. The MIC for VOR was $0.5 \mu \mathrm{g} / \mathrm{ml}$ for the isolate of A. fumigatus and $0.25 \mu \mathrm{g} /$ $\mathrm{ml}$ for both isolates of $A$. flavus and A. niger. The MEC for CAS was $0.06 \mu \mathrm{g} / \mathrm{ml}$ for both isolates of $A$. fumigatus and A. flavus, and $0.03 \mu \mathrm{g} / \mathrm{ml}$ for the isolate of A. niger. FICI indices ranged from 0.25 to 0.50 , indicating the combination of VOR and CAS synergy for each strain.

\section{Survival}

Survival was prolonged among the rats treated with the combination of VRC and CAS for all the strains (Fig. 1.). The survival of rats in the combined therapy groups was significantly improved compared to the CAS monotherapy for the three strains tested $(P<0.05)$, and compared to VRC alone for the strains of A. flavus and A. niger $(P<0.05)$. Even though the survival rate of the combined therapy is higher than the VRC monotherapy for the strain of $A$. fumigatus, there were no significant differences $(P>0.05)$.

\section{Serum Galactomannan}

The serum galactomannan levels were significantly lower in the rats combined therapy of VRC and CAS in comparison with the other groups for all the strains $(P<0.05)$ (Fig. 2). There was no difference in VRC groups and CAS groups in comparison with the control 

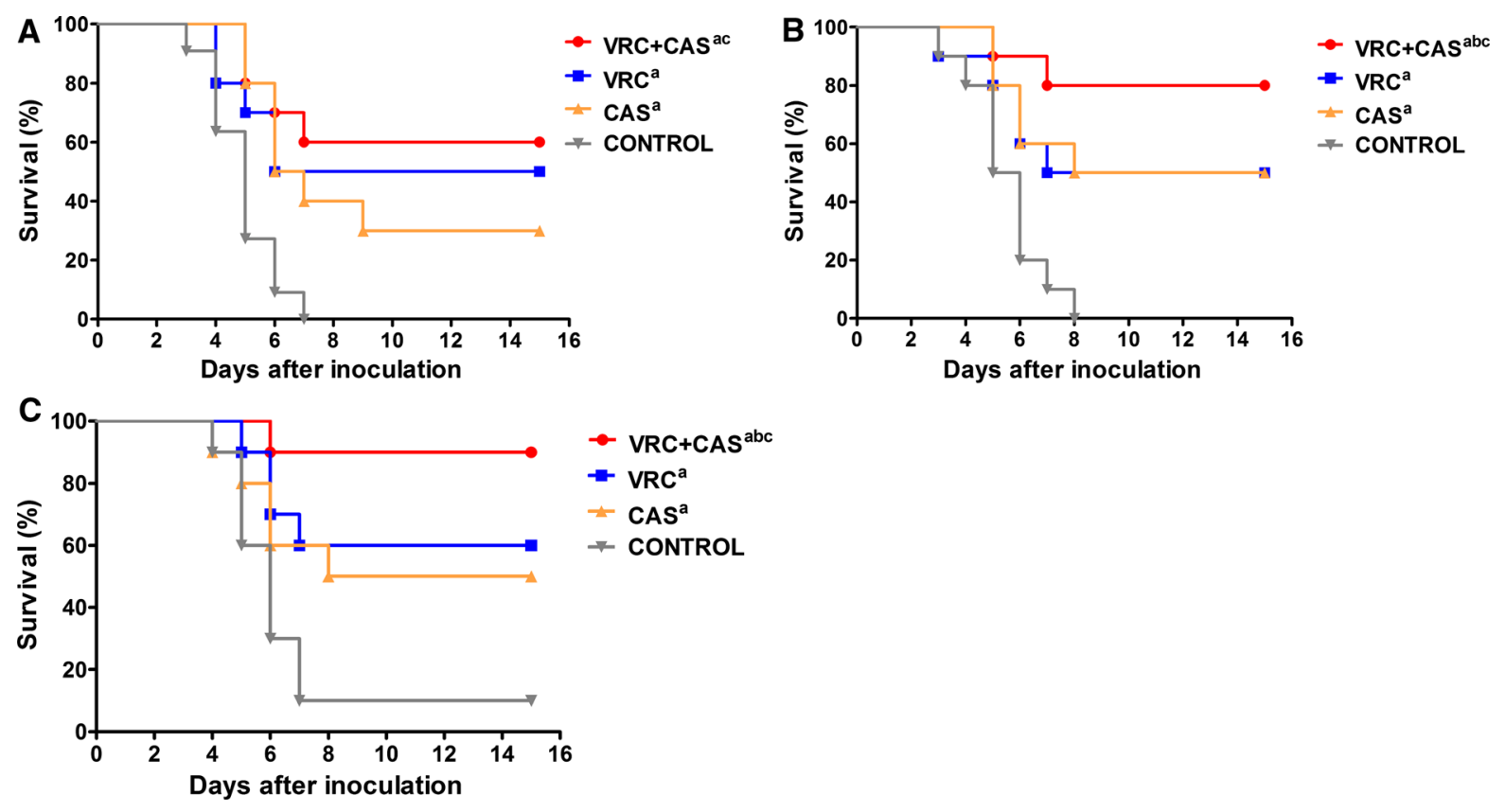

Fig. 1 Survival of rats infected with $1 \times 10^{8}$ conidia of A.fumigatus (a), A. flavus (b) and A. niger (c). VRC, at $1 \mathrm{mg} / \mathrm{kg} /$ day intravenous. All treatments began on day 1 postinfection and continued for 10 days. a $P<0.05$ versus voriconazole at $10 \mathrm{mg} / \mathrm{kg}$ q12-h intravenous; CAS, caspofungin

CONTROL; b $P<0.05$ versus VRC; c $P<0.05$ versus CAS

Fig. 2 Expression of galactomannan in rats infected by A. fumigatus (a), A. flavus (b) and A. niger (c). Animals treated with the VRC and CAS combination had significantly lower levels of galactomannan in comparison with all other groups $(*, P<0.05)$. VRC, voriconazole at $10 \mathrm{mg} / \mathrm{kg}$ q12-h intravenous; CAS, caspofungin at $1 \mathrm{mg} / \mathrm{kg} / \mathrm{day}$ intravenous
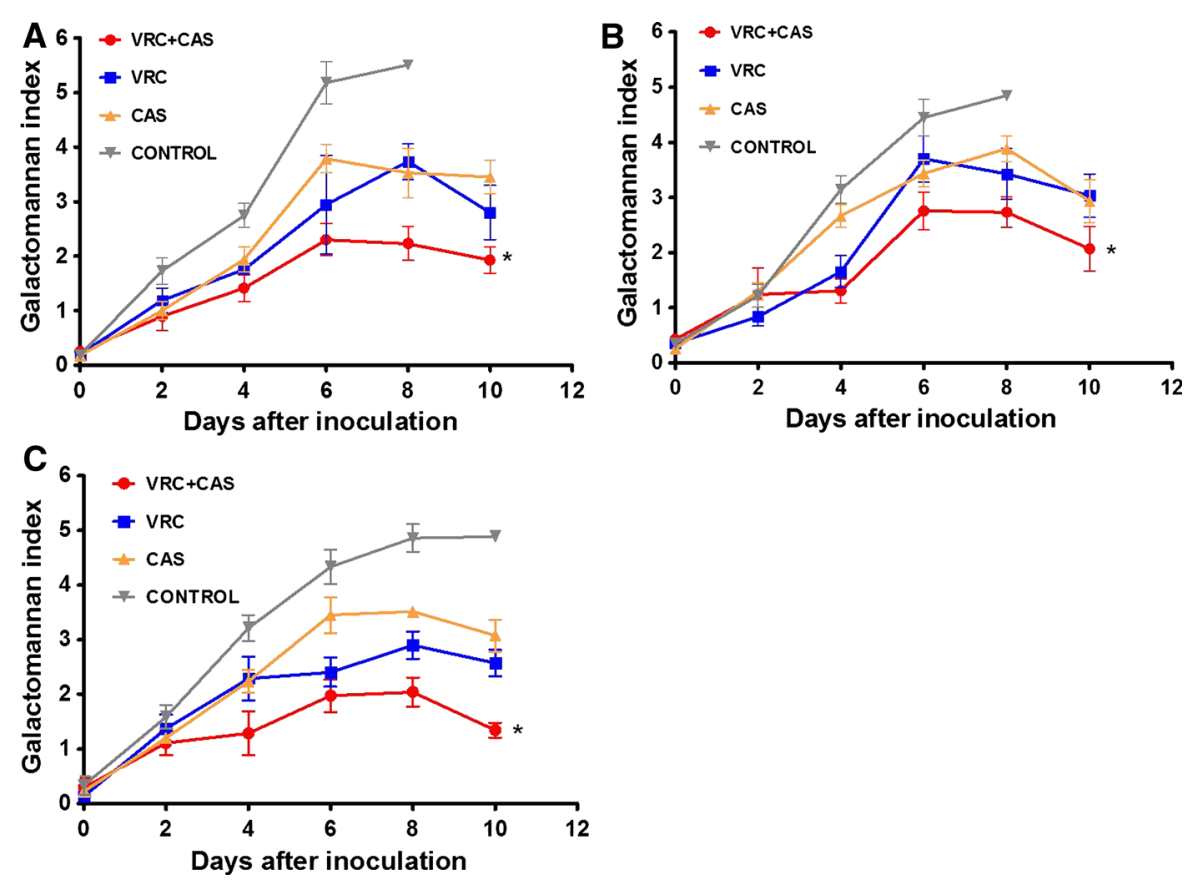

Residual Fungal Burden

The results of the pulmonary tissue residual fungal burden are shown in Fig. 3. There was significant 
Fig. 3 The mean pulmonary tissue residual fungal burden ( $\log \mathrm{CFU} / \mathrm{g}$ ) in rats infected with $\mathrm{A}$. fumigatus (a), A. flavus (b) and $A$. niger (c) on day 5 after challenge. $V R C$, voriconazole at $10 \mathrm{mg} / \mathrm{kg}$ q12 h intravenous; $C A S$, caspofungin at $1 \mathrm{mg} / \mathrm{kg} / \mathrm{day}$ intravenous. $a P<0.05$ versus CONTROL; $b P<0.05$ versus VRC; $c$ $P<0.05$ versus CAS
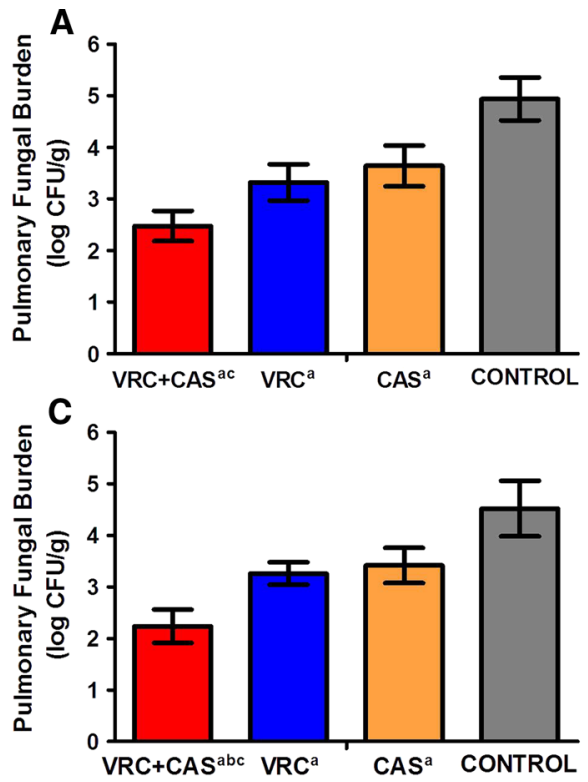

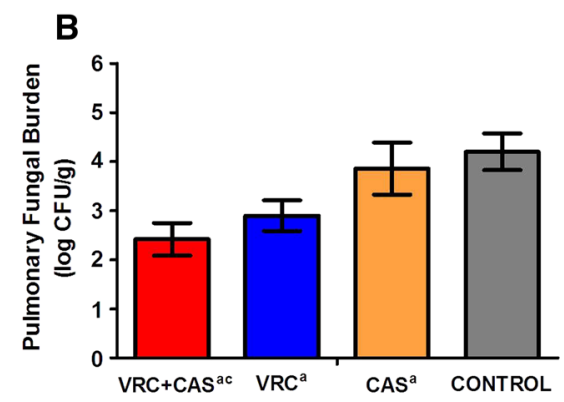

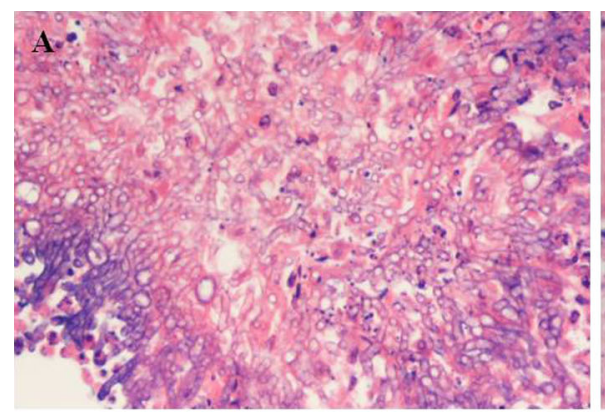
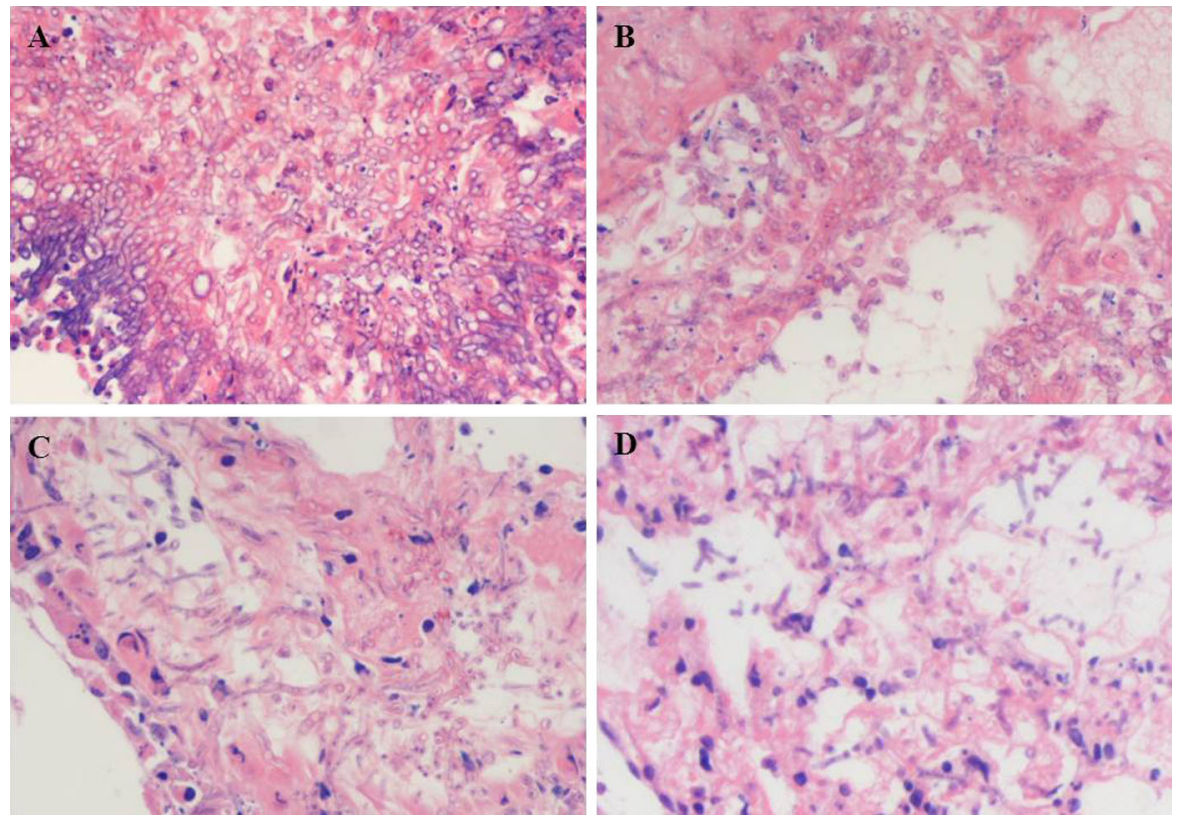

Fig. 4 The histological studies in rats infected with A. fumigatus (a), A. flavus (b), and A. niger (c) on day 5 after challenge. a Lung sections of control rat infected with the $A$. fumigatus; HE $\times 200$. b Lung section of a rat infected with the

A. fumigatus treated with CAS; HE $\times 200$. c Lung section of a rat infected with the A. fumigatus treated with VRC; $\mathrm{HE} \times 200$. d Lung section of a rat infected with the A. fumigatus treated with VRC and CAS; HE $\times 200$

reduction of residual fungal burden (CFU/g) in the combination of VRC- and CAS-treated rats, compared with the effect of CAS alone, or no treatment for all the strains $(P<0.05)$. The combined therapy worked better

than VRC in only the strain of $A$. niger $(P<0.05)$. There were no significant differences in the combination rats in comparison with that of VRC alone for the strain of A. fumigatus and A. flavus $(P>0.05)$. 
Histopathology

The histological features of aspergillosis were studied in the lungs of rats in all treatment groups (Fig. 4). Histological studies of untreated control rats showed dense clumps of hyphae and demonstrated a typical acute angle branching septate hyphae A. fumigatus. Rats treated with CAS showed alveolar collapse, inflammatory infiltration, and signs of necrosis, which were relatively mild in rats treat with VRC and the combination of VRC and CAS.

\section{Discussion}

In this study, we investigated the efficacy of the combination of VRC and CAS compared to both monotherapies in vitro and in vivo using an experimental model of IPA infected by A. fumigatus, A. flavus, or A. niger. The combination of VRC and CAS demonstrated significantly enhanced efficacy for the strain of A. flavus and A. niger. We observed an synergistic interaction in vitro for each strain,a prolongation of survival,a significant reduction in serum galactomannan levels, and residual fungal burden $(\mathrm{CFU} / \mathrm{g})$ in combination of VRC and CAS compared with drug alone or no treatment.

To our knowledge, this is the first study to evaluate the therapy efficacy of the echinocandin-triazole combination in transiently neutropenic rats infected by A. fumigatus, A. flavus, or A. niger as measured by the prolongation of survival, reduction pulmonary tissue residual fungal burden, decrease in serum galactomannan levels, and histological studies. However, the dose of VRC or CAS used and the species of Aspergillus assayed in this study were single, which were possible limitation of the experimental design. For the VRC, metabolized faster in rodents than in humans, the area under the concentration-time curve (AUC)/MIC ratio is considered the pharmacokinetic/ pharmacodynamic (PK/PD) index determining therapeutic efficacy $[10,18]$. For the echinocandins, such as CAS,both the AUC/MIC and the $C_{\max } / \mathrm{MIC}$ are considered the PK/PD index predicting efficacy [10, 12]. Van de Sande et al. [10] elucidated the pharmacokinetics of VOR in rats model of IPA and found that the dosage of VRC at $10 \mathrm{mg} / \mathrm{kg}$ q12 $\mathrm{h}$ showed excellent efficacy, without toxic side effects in renal and hepatic functions. Meanwhile, several studies showed that the dose of CAS at $1 \mathrm{mg} / \mathrm{kg} / \mathrm{d}$ has a better therapeutic effect in treatment IPA [12, 16, 17]. So, there was only a single dose of VRC or CAS used in this study. Though, as previously described [7, 10, 11, 13], we used one sensitive isolate of each of the species of Aspergillus assayed, this may be a limitation of the study.

There are contradictory results in the previous studies VRC combined echinocandin treatment for invasive aspergillosis (IA). Van de Sande et al. [10], in a neutropenic rat model of IPA by A. fumigatus, found combining of VRC and anidulafungin (AFG) does not significantly prolong the survival compared to the VOR monotherapy $(P=0.3290)$. Petraitis et al. [13] demonstrated that the survival of persistently neutropenic rabbits of IPA by $A$. fumigatus treatment with VRC $(10 \mathrm{mg} / \mathrm{kg}$ every $8 \mathrm{~h})$ combined with AFG at $5 \mathrm{mg} / \mathrm{kg} /$ day was $60 \%$, but $27 \%$ for the VRC + AFG $(10 \mathrm{mg} / \mathrm{kg} /$ day $)$ group $(P<0.001)$. It suggested that AFG at a dosage of $5 \mathrm{mg} / \mathrm{kg} /$ day was synergistic but antagonistic at $10 \mathrm{mg} / \mathrm{kg} / \mathrm{day}$. In our study, combining both agents worked better than CAS alone in each strains and better than VRC in the strains of A. flavus and A. niger. Similarly, the therapy with VRC and CAS combined in guinea pig models of IA by $A$. fumigatus significantly improved survival and achieved significant reduction in residual fungal burden compared to respective monotherapies [16]. In other studies [15], the combined treatment of VRC and AFG in a murine IA model infected by A. flavus was found to significantly improve the survival, reduce the fungal burden and the galactomannan levels in comparison with AFG alone, but significantly improved only in a few cases compared to VRC alone. In the in vitro studies, our results showed each Aspergillus was susceptible for VRC and AFG and synergistic effect of VRC combined with AFG. Seyedmousavi et al. [23], in a non-neutropenic murine model of IA by VRC-susceptible or VRC-resistant $A$. fumigatus, demonstrated that the combination of VRC and AFG was synergistic in VRC-susceptible IA, but additive in VRC-resistant IA. Comparison with the previous studies, there are certain discrepancies. These might be due to the deviation of the type and number of animals, infection route and dose, therapeutic dosage, and so on.

Our results demonstrated VRC monotherapy is more therapeutically effective than the use of CAS in the rats of IPA by A. fumigatus. Combination of VRC and CAS 
does not significantly improve the survival in the treatment for experimental IPA by A. fumigatus. While in the rats of IPA by A. flavus or A. niger, our data confirm the therapy of VRC and CAS significantly improve the therapeutic outcome compared with drug alone. It suggests that the efficacy of the combination is different between Aspergillus species and the efficacy in A. flavus and A. niger is better than in A. fumigatus. However, there are some studies of combination therapy for IPA by A. fumigatus [7, 10, 12-14], more rarely by $A$. flavus $[15,27]$ and $A$. niger $[27,28]$. The therapeutic outcome of the studies or cases of IPA by $A$. flavus or A. niger are generally successful.

In conclusion, there is a synergistic effect of the combination of VRC and CAS in the treatment for IPA by $A$. flavus and A. niger. Meanwhile, our data indicate that the echinocandin-triazole combination in comparison with single agents may confer a small efficacy benefits in the treatment for IPA by A. fumigatus. The efficacy of the echinocandin-triazole combination for IPA by A. fumigatus, A. flavus, and A. niger needs further clinical studies.

Acknowledgments This work was supported by grants from the National Natural Science Foundation of China (Grant Nos. 81270064, 81200063).

Conflict of interest There are no conflicts of interest for this article.

Open Access This article is distributed under the terms of the Creative Commons Attribution License which permits any use, distribution, and reproduction in any medium, provided the original author(s) and the source are credited.

\section{References}

1. Snelders E, Melchers WJ, Verweij PE. Azole resistance in Aspergillus fumigatus: a new challenge in the management of invasive aspergillosis? Future Microbiol. 2011;6:335-47.

2. Perfect J, Cox G, Lee J, Kauffman C, De Repentigny L, Chapman S, et al. The impact of culture isolation of Aspergillus species: a hospital-based survey of aspergillosis. Clin Infect Dis. 2001;33:1824-33.

3. Marr KA. Fungal infections in hematopoietic stem cell transplant recipients. Med Mycol. 2008;46:293-302.

4. Walsh TJ, Anaissie EJ, Denning DW, Herbrecht R, Kontoyiannis DP, Marr KA, et al. Treatment of aspergillosis: clinical practice guidelines of the Infectious Diseases Society of America. Clin Infect Dis. 2008;46:327-60.

5. Zmeili O, Soubani A. Pulmonary aspergillosis: a clinical update. QJM. 2007;100:317-34.
6. Greene RE, Mauskopf J, Roberts CS, Zyczynski T, Schlamm HT. Comparative cost-effectiveness of voriconazole and amphotericin B in treatment of invasive pulmonary aspergillosis. Am J Health-Syst Pharm. 2007;64:2561-8.

7. Petraitis V, Petraitiene R, Sarafandi AA, Kelaher AM, Lyman CA, Casler HE, et al. Combination therapy in treatment of experimental pulmonary aspergillosis: synergistic interaction between an antifungal triazole and an echinocandin. J Infect Dis. 2003;187:1834-43.

8. Luque JC, Clemons KV, Stevens DA. Efficacy of micafungin alone or in combination against systemic murine aspergillosis. Antimicrob Agents Chemother. 2003;47:1452-5.

9. Kirkpatrick WR, Perea S, Coco BJ, Patterson TF. Efficacy of caspofungin alone and in combination with voriconazole in a guinea pig model of invasive aspergillosis. Antimicrob Agents Chemother. 2002;46:2564-8.

10. van de Sande WW, Mathot RA, Marian T, van Vianen W, Tavakol M, Rijnders BJ, et al. Combination therapy of advanced invasive pulmonary aspergillosis in transiently neutropenic rats using human pharmacokinetic equivalent doses of voriconazole and anidulafungin. Antimicrob Agents Chemother. 2009;53:2005-13.

11. Clemons KV, Stevens DA. Efficacy of micafungin alone or in combination against experimental pulmonary aspergillosis. Med Mycol. 2006;44:69-73.

12. Wagner C, Graninger W, Presterl E, Joukhadar C. The echinocandins: comparison of their pharmacokinetics, pharmacodynamics and clinical applications. Pharmacology. 2006;78:161-77.

13. Petraitis V, Petraitiene R, Hope WW, Meletiadis J, Mickiene D, Hughes JE, et al. Combination therapy in treatment of experimental pulmonary aspergillosis: in vitro and in vivo correlations of the concentration-and dose-dependent interactions between anidulafungin and voriconazole by Bliss independence drug interaction analysis. Antimicrob Agents Chemother. 2009;53:2382-91.

14. Marr KA, Schlamm H, Rottinghaus ST, Jagannatha S, Bow EJ, Wingard JR, et al. A randomised, double-blind study of combination antifungal therapy with voriconazole and anidulafungin versus voriconazole monotherapy for primary treatment of invasive aspergillosis. Mortality. 2012;27:0868.

15. Calvo E, Pastor FJ, Salas V, Mayayo E, Guarro J. Combined Therapy of Voriconazole and Anidulafungin in Murine Infections by Aspergillus flavus. Mycopathologia. 2012;173:251-7.

16. MacCallum DM, Whyte JA, Odds FC. Efficacy of caspofungin and voriconazole combinations in experimental aspergillosis. Antimicrob Agents Chemother. 2005;49:3697-701.

17. Hope W, Drusano G. Antifungal pharmacokinetics and pharmacodynamics: bridging from the bench to bedside. Clin Microbiol Infect. 2009;15:602-12.

18. Levêque D, Nivoix Y, Jehl F, Herbrecht R. Clinical pharmacokinetics of voriconazole. Int $\mathrm{J}$ Antimicrob Agents. 2006;27:274-84.

19. Rex JH. Reference method for broth dilution antifungal susceptibility testing of filamentous fungi: Approved Standard: Clinical and Laboratory Standards Institute; 2008.

20. Dannaoui E, Lortholary O, Dromer F. In vitro evaluation of double and triple combinations of antifungal drugs against Aspergillus fumigatus and Aspergillus terreus. Antimicrob Agents Chemother. 2004;48:970-8. 
21. Krishnan-Natesan S, Wenjuan W, Chandrasekar PH. In vitro efficacy of the combination of voriconazole and anidulafungin against voriconazole-resistant cyp51A mutants of Aspergillus fumigatus. Diagn Microbiol Infect Dis. 2012;73:135-7.

22. Cuenca-Estrella M, Gomez-Lopez A, Garcia-Effron G, Alcazar-Fuoli L, Mellado E, Buitrago MJ, et al. Combined activity in vitro of caspofungin, amphotericin $\mathrm{B}$, and azole agents against itraconazole-resistant clinical isolates of Aspergillus fumigatus. Antimicrob Agents Chemother. 2005;49:1232-5.

23. Seyedmousavi S, Brüggemann RJ, Melchers WJ, Rijs AJ, Verweij PE, Mouton JW. Efficacy and pharmacodynamics of voriconazole combined with anidulafungin in azoleresistant invasive aspergillosis. J Antimicrob Chemother. 2013;68:385-93.

24. Krishnan-Natesan S, Wu W, Chandrasekar PH. In vitro efficacy of the combination of voriconazole and anidulafungin against voriconazole-resistant cyp 51A mutants of
Aspergillus fumigatus. Diagn Microbiol Infect Dis. 2012;73:135-7.

25. Zhao Y, Park S, Warn P, Shrief R, Harrison E, Perlin DS. Detection of Aspergillus fumigatus in a rat model of invasive pulmonary aspergillosis by real-time nucleic acid sequence-based amplification. $J$ Clin Microbiol. 2010;48:1378-83.

26. Giudice PL, Campo S, Verdoliva A, Rivieccio V, Borsini F, De Santis R, et al. Efficacy of PTX3 in a rat model of invasive aspergillosis. Antimicrob Agents Chemother. 2010;54:4513-5.

27. Singh N, Limaye AP, Forrest G, Safdar N, Munoz P, Pursell $\mathrm{K}$, et al. Combination of voriconazole and caspofungin as primary therapy for invasive aspergillosis in solid organ transplant recipients: a prospective, multicenter, observational study. Transplantation. 2006;81:320-6.

28. Person AK, Chudgar SM, Norton BL, Tong BC, Stout JE. Aspergillus niger: an unusual cause of invasive pulmonary aspergillosis. J Med Microbiol. 2010;59:834-8. 